\title{
Tumor Budding as a Useful Prognostic Indicator in Early Oral Squamous Cell Carcinoma
}

Tadaaki Kirita*, Nobuhiro Yamakawa, Nobuhiro Ueda and Takahiro Yagyuu

Department of Oral and Maxillofacial Surgery, Nara Medical University, Schijo-cho, Nara-Kashihara, Japan

\begin{abstract}
Tumor budding, defined as the presence of single cancer cells or small clusters of fewer than five cells at the tumor invasive front, has been reported to be an independent prognostic factor for several cancers together with margin status, depth of invasion, lymph vascular invasion, and lymph node metastasis. Distant metastasis and poor prognosis were significantly more common in the higher budding group. In this short review, we focused on the studies related to tumor budding in several cancers including oral squamous cell carcinoma(OSCC) and showed the association among the intensity of tumor budding, risk of lymph node metastasis, and poor prognosis in OSCC is strongly supported by many studies. Moreover, arguments for the assessment of tumor budding, scoring system, stains to mark budding, specimens, and the association with epithelial mesenchymal transition (EMT) are also discussed.
\end{abstract}

Keywords: Tumor budding; Oral cavity; Squamous cell carcinoma; Prognostic indicator

\section{Introduction}

The oral and pharyngeal region is the sixth leading site of cancer in the world, and oral cancer is widely accepted to have a higher incidence in people older than 50 years, primarily because of the relationship with chronic exposure to tobacco, alcohol, and other carcinogenic products. In particular, oral cancer is most common in India, Bangladesh, Pakistan, and Sri Lanka, and accounts for about one-third of all cancers [1]. The incidence of this cancer in young adults (age: $<40$ years) appears to be increasing in many Western countries [2].

In general, small and superficial carcinoma of the oral cavity are usually amenable to surgical resection or radiotherapy. Therefore, use of a single modality, most commonly surgery, is selected as the definitive treatment in early stage cancer of the oral cavity. However, some patients with early oral squamous cell carcinoma (OSCC) who were considered curable eventually suffer from locoregional failure after definitive surgery. These oral cavity cancers represent a heterogeneous group of patients who may be at high risk of locoregional recurrence. In order to prevent relapse, several novel interventions are starting to be performed based on new basic research and clinical data. Several histopathological prognostic indicators of oral cancer have been reported recently, such as tumor grade, mode of invasion, pattern of invasion, lymphovascular invasion, perineural invasion, depth of invasion, extracapsular lymph node invasion, and resection margin status [3,4]. Although these are important to select the initial treatment, adjuvant therapy, and to follow up, some are controversial and have limited evidence.

Recently, the utility and significance of tumor budding in histopathological examinations were shown for several early stage cancers such as colon, oesophageal, and gastric cancer. Tumor budding, defined as the presence of single cancer cells or small clusters of fewer than five cells at the invasive front (IF), has been reported to be an independent prognostic factor for lung and T1 stage oesophageal squamous cell carcinomas together with lymphatic and venous invasion. In addition, lymph node metastasis is significantly more common in the higher budding group $[5,6]$. In colorectal cancers, Prall et al. investigated budding in a series of stage I and II colorectal cancer patients and found that tumors with high budding had a higher risk of relapse and distant metastasis [7].
In this short review, we focus on the studies related to tumor budding in several carcinomas including OSCC and identify whether tumor budding really has an impact on the locoregional recurrence and prognosis of early OSCC, then, present the prognostic value of tumor budding in early OSCC.

\section{Literature Review}

Tumor budding was initially designated as tumor sprouting to the stroma and was first reported in 1954 by Imai, who described it as a morphological feature along the invasive margin reflecting more active tumor growth in several human cancers [8]. In 1961, Mc Gavran, in a study of squamous cell carcinoma of the larynx, showed that there was a significant correlation between the frequency of metastasis and the types of invasive growth patterns. Two distinct types were identified - one with a "pushing" and the other an "infiltrating" margin [9]. Afterwards, many authors have reported a close correlation the histologic malignancy scores for the pattern of invasion (POI) in head and neck squamous cell carcinoma and the outcome of the disease $[10,11]$, but other investigators have found no significant relationship [12] and limited value for prognosis. Attramadal [13] reported that POI correlated significantly with tumor budding, but budding was a stronger prognosticator in OSCC. There are some reasons behind this variability, such as a lack of objectivity and difficulty in judgement. An important refinement of POI, known as tumor budding, was defined with general agreement as the presence of individual cancer cells or small clusters (fewer than five cancer cells) at the invasive front (IF) of the tumor, and is considered the first step in the metastases of a solid tumor [6] (Figure 1). Currently, this definition is generally used worldwide by most pathologists.

*Corresponding author: Tadaaki Kirita, Professor and Chair, Department of Ora and Maxillofacial Surgery, Nara Medical University, 840 Schijo-cho, Nara-Kashihara 634-8521, Japan, Tel: +81-744-22-3051; Fax: +81-744-29-8875; E-mail: tkirita@naramed-u.ac.jp

Received May 10, 2018; Accepted June 20, 2018; Published June 22, 2018

Citation: Kirita T, Yamakawa N, Ueda N, Yagyuu T (2018) Tumor Budding as a Useful Prognostic Indicator in Early Oral Squamous Cell Carcinoma. J Cancer Sci Ther 10: 162-167. doi: 10.4172/1948-5956.1000536

Copyright: (c) 2018 Kirita T, et al. This is an open-access article distributed under the terms of the Creative Commons Attribution License, which permits unrestricted use, distribution, and reproduction in any medium, provided the original author and source are credited. 


\section{Assessment of tumor budding and its prognostic value in several cancers}

The definite implementation of tumor budding assessment depends on a selected, internationally accepted, scoring system. However, scoring systems of tumor budding are different in reports on several cancers. In colorectal cancer, Hase [14] first divided tumor budding into two groups according to degree: none or mild (BD-1) and moderate or severe (BD-2). Classification of lesions as BD-1 or BD-2 was based on the predominant pattern of tumor budding in the resected specimen and patients with BD-2 lesions had significantly higher recurrence rates and lower 5-year and 10-year survival rates than those in the BD-1 group. Ueno [15] used a more quantitative method to assess the degree of budding. At the invasive front region after selecting a field in which "budding" intensity was considered maximal, the number of "budding" foci were counted in a field measuring $0.385 \mathrm{~mm}^{2}$ using a $25 \mathrm{x}$ objective lens. More than 10 budding cells was set as the cut-off score for high-grade "budding" and was associated with lower 5-year survival rates than that of low-grade "budding". Multivariate analysis showed that tumor budding was selected as the significant independent variable, together with the number of nodes involved, extramural spread, lymphocytic infiltration, apical nodal involvement, and tumor differentiation. In 2016, the International Tumor Budding Consensus Conference (ITBCC) [16] showed that tumor budding is a wellestablished independent prognostic factor and reached agreement on an evidence-based, standardized scoring system in colorectal cancer. ITBCC guidelines defined tumor budding as a single tumor cell or a cell cluster consisting of four tumor cells or less; tumor budding is an independent predictor of lymph node metastases in pT1 and survival in stage II colorectal cancer; tumor budding is counted on hematoxylin and eosin; tumor budding is assessed in one hot spot (in a field measuring $0.785 \mathrm{~mm}^{2}$ ) at the invasive front; and a three-tier system should be used along with budding count ( $0-4$ buds: low, 5-9 buds: intermediate, 10 or more buds: high) to facilitate risk stratification. Some studies have used the average score of multiple fields ( 5 or 10 high-power fields) to score tumor budding, and this increased scoring reproducibility because selection of a single field with the highest density of tumor budding may be subject to interobserver variation $[17,18]$. On the other hand, counting multiple fields may "dilute" the final (mean) tumor bud count in cases with many focal tumor buds. The "hot spot" method, therefore, better reflects the maximal extent of tumor budding at the invasive front. The ITBCC group recommends the use of the "hot spot" method and to ensure that the field with the highest tumor budding is selected, it was recommended that 10 separate fields (20x objective) along the invasive front are scanned before counting tumor buds in the single field selected as the "hot spot" [16].

In esophageal cancer, tumor budding is defined similarly to colorectal cancer - tumor buds are isolated single cancer cells or microscopic clusters of undifferentiated cancer cells composed of fewer than five cancer cells found outside the invasive margin of the tumor [19]. On the scoring system, budding intensity was considered maximal in the slide containing the deepest tumor penetration, and the number of budding foci was counted using a 20x objective lens ("hotspot" method) $[6,19]$. In some studies, the cut-off point for risk grade was five buds (low grade: $<5$ buds, high grade $\geq 5$ buds), and in others, the cut-off point was three buds (rare: $<3$ buds, frequent: $\geq 3$ buds). A single study classified tumors into two grades as only bud-positive or budnegative (positive: distinct budding at the invasive front, negative: no budding at the invasive front) [20]. In all studies, tumor budding in esophageal cancer was also a significant predictive factor for OSCC, and cases with high-grade budding had a poorer outcome than cases with low grade budding using different scoring systems. In oral and head and neck squamous cell carcinomas, tumor budding is defined similarly to colorectal, esophageal, and other epithelial cancers. Therefore, there is general agreement in defining tumor budding as the presence of either isolated single cells or small cell clusters comprising fewer than five cells at the invasive tumor front. By contrast, there is no consensus cut-off point for risk grade for the number of tumor buds even in oral and head and neck squamous cell carcinoma. In spite of this, a cut-off points of five in one high-power field (40x) (low grade: low grade: $<5$ buds, high grade $\geq 5$ buds) is widely accepted by many investigators [21-23]. Different scoring methods have been employed in several studies, but they can be summarized by two major approaches: one is the "hot spot" method that analyzes only one high-power field (40x) and the other is the " 10 high-power fields" method that averages the 10 most dense highpower fields (20x or 40x), in which budding cells from high-power fields were detected after scanning the whole length of the invasive front at low magnification. The " 10 high-power fields" method was proposed in colorectal cancer $[17,24]$ which reported high inter- and intra-observer agreement using this method. However, the ITBCC group recommends the use of the "hot spot" method in colorectal cancer, and further studies are needed to understand the prognostic ability and reproducibility of these methods in oral and head and neck squamous cell carcinomas. Moreover, there is a loss of unity in "high-power fields" for scoring, where objective magnifications of 20x or 40x are usually used. There are still some variations in scoring, and consensus is needed for appropriate comparison of results, even for oral and head and neck squamous cell carcinomas. Many studies have reported that a high tumor budding score at the invasive front is strongly associated with cell proliferation, lymph node metastasis including occult metastasis and disease-specific mortality in OSCC, especially in T1/2 stage OSCC [21-26]. The number of included cases, tumor stages, specimens, risk stratification cut-off point and prognostic value of tumor budding in previous studies are summarized in Table 1. Moreover, it is reported that the progression level of extracapsular spread and tumor budding for cervical lymph node metastasis of OSCC correlated with locoregional recurrence and distant metastasis, and the tumor budding was identified as an independent prognostic factor in a multivariate analysis [27]. These findings suggest that despite the lack of a standardized scoring system, tumor budding is a reliable marker of tumor progression and bad outcomes independent of the evaluation system used.

Recently, Weichert [28] introduced a novel grading scheme that was highly prognostic for patient survival (OS, DFS, and DSS): cell nest size (CNS) and tumor budding activity (BA) in lung squamous cell carcinoma. Boxberg [29] conducted an analysis of this grading scheme in a cohort of 157 patients with OSCC and showed that, analogous to SQCC-L, a simple grading scheme incorporating the two most prognostic histomorphological characteristics, BA and CNS, can be used successfully in OSCC. In their report, budding was assessed in areas with maximal BA and was scored separately in one high-power field (HPF; 40x) displaying the highest BA and 10 HPFs. In one HPF, low BA was defined as 1-4 budding nests and high BA as $\geq 5$ budding nests. In 10 HPFs, no budding was scored as 1, low BA and 1-14 buds was scored as 2 , and high BA and $\geq 15$ budding nests was scored as 3 . Cell nests were defined as clustered tumor cells surrounded by stroma and were classified based on the size of the smallest invasive cell nest. Clusters of $>15$ tumor cells were classified as large cell nests (score 1), 5-15 tumor cells as intermediate cell nests (score 2), 2-4 tumor cells as small cell nests (score 3 ), and single cell invasion was stated for individual tumor cells (score 4). CNS was assessed at the invasive margin and within the tumor core region. The grading score was 


\begin{tabular}{|c|c|c|c|c|c|c|c|c|c|}
\hline Authors & Year & Location & $\begin{array}{c}\text { Number } \\
\text { of } \\
\text { cases }\end{array}$ & Stage & Specimen & Cut-off & $\begin{array}{l}\text { Objective } \\
\text { lens }\end{array}$ & Immunohistochemistry & Main findings \\
\hline $\begin{array}{l}\text { Sarioglu et al. } \\
\text { [49] }\end{array}$ & 2010 & Larynx & 64 & II-IV & -- & $\begin{array}{l}\text { buds at } \\
2 / 3 \text { of IF }\end{array}$ & -- & - & TB is a prognostic marker for DM \\
\hline Wang et al. [22] & 2011 & Tongue & 230 & I-IV & Resection & 5 buds & $20 x$ & - & TB correlates with poor prognosis and EMT \\
\hline Luo et al. [50] & 2012 & Nasopharynx & 105 & I-IV & Resection & 5 buds & $400 x^{*}$ & Cytokeratin & TB was associated with poor prognosis \\
\hline $\begin{array}{l}\text { Almangush et } \\
\text { al. [23] }\end{array}$ & 2014 & Tongue & 233 & I-II & Resection & 5 buds & $20 x$ & - & $\begin{array}{l}\text { TB and TD are associated with poor } \\
\text { prognosis }\end{array}$ \\
\hline $\begin{array}{l}\text { Marangon et al. } \\
{[21]}\end{array}$ & 2014 & Oral & 57 & - & Resection & 5 buds & $20 x$ & Cytokeratin & $\begin{array}{c}\text { Laminin-5 } \square 2 \text { expression is associated with } \\
\text { high-intensity of TB }\end{array}$ \\
\hline $\begin{array}{l}\text { Almangush et } \\
\text { al. [36] }\end{array}$ & 2015 & Tongue & 311 & $I-I I$ & Resection & 5 buds & $20 x$ & Cytokeratin & TB was associated with poor prognosis \\
\hline Xie et al. [25] & 2015 & Tongue & 195 & I-II & Resection & 5 buds & $20 x$ & Cytokeratin & $\begin{array}{l}\text { TB correlates with occult cervical LNM and } \\
\text { poor prognosis }\end{array}$ \\
\hline Angadi et al. [51] & 2015 & Oral & 75 & - & Resection & 10 buds & $\begin{array}{l}20 x \times 5 \\
\text { fields }\end{array}$ & - & TB is a predictor for LNM \\
\hline $\begin{array}{c}\text { Sawazaki-Calone } \\
\text { et al. [52] }\end{array}$ & 2015 & Oral & 113 & - & Resection & 5 buds & $20 x$ & - & BD was associated with poor prognosis \\
\hline Jensen et al. [53] & 2015 & Oral & 199 & - & Resection & - & - & Cytokeratin & TB correlates with LNM and poor prognosis \\
\hline $\begin{array}{l}\text { Attramadal et } \\
\text { al. [13] }\end{array}$ & 2015 & Oral & 392 & I-II & Resection & 5 buds & $20 x$ & Cytokeratin & TB correlates with poor prognosis \\
\hline Seki et al. [39] & 2016 & Tongue/FOM & 91 & I-IV & Biopsy & 3 buds & $20 x$ & Cytokeratin & $\begin{array}{c}\text { TB is an independent predictor unaffected by } \\
\text { the status of infiltrative patterns and tumour } \\
\text { depth for LNM }\end{array}$ \\
\hline $\begin{array}{l}\text { Boxberg et al. } \\
{[29]}\end{array}$ & 2017 & Oral & 157 & I-IV & Resection & - & $\begin{array}{l}40 x^{* *} \times 10 \\
\text { fields }\end{array}$ & - & $\begin{array}{c}\text { TB and CNS are associated with poor } \\
\text { prognosis }\end{array}$ \\
\hline Seki et al. [40] & 2017 & Oral & 209 & I-IV & Biopsy & $\begin{array}{l}3 \text { and } 5 \\
\text { buds }\end{array}$ & $20 x$ & Cytokeratin & $\begin{array}{l}\text { TB using a cut-off value } \geq 3 \text { is a predictor for } \\
\text { LNM and poor prognosis, especially in } \mathrm{T} 1 / 2 \\
\text { stages and cNO patients }\end{array}$ \\
\hline $\begin{array}{l}\text { Pedersen, et al. } \\
{[54]}\end{array}$ & 2017 & Oral & 222 & I-IV & Resection & $\begin{array}{l}920 \\
\mu \mathrm{m} 2 / 6 \\
\text { buds }\end{array}$ & - & Cytokeratin & $\begin{array}{l}\text { High DTBC was independent predictor of } \\
\text { both OS and PFS }\end{array}$ \\
\hline
\end{tabular}

TB: Tumour Budding; EMT: Epithelial-Mesenchymal Transition; FOM: Floor of Mouth; TD: Tumour Depth; LN: Lymph Node Metastasis; DM: Distant Metastasis; BD: Tumor Budding and Depth of Invasion; CNS: Cell Nest Size; DTBC: Digital Tumor Bud Count; OS: Overall Survival; PFS: Progression Free Survival. * A microscopic Tumor Budding and Depth of Invasion; CNS: Cell
field of $400 x$ magnification, ${ }^{* *}$ High-power field

Table 1: Summary of previous published studies of tumor budding in oral and head and neck squamous cell carcinoma

summed for these two variables and ranged from 2 to 7 . Tumors with score 2-3 were defined as Grade1, score 4-6 as Grade 2, and score 7 as Grade3. Multivariate survival analysis confirmed that the prognostic impact of this grading scheme was independent of clinicopathological parameters (e.g., differentiation, age, sex, and stage). This novel grading system is interesting and attractive because a combination of these two factors represents the malignant potential of a given carcinoma more reliability than each individual parameter alone, not only for lung squamous cell carcinoma, but also for OSCC [28]. However, further prospective and retrospective studies are needed to confirm these results.

\section{Immunohistochemical staining or H\&E staining on assessment of tumor budding}

To assess the degree of tumor budding, Prall [30] were the first to use pan-cytokeratin staining to mark budding cells at the invasive margin in colorectal cancer in 2005. They showed that immunohistochemical highlighting of budding cells by pan-cytokeratin antibodies may have contributed to a significant increase in budding-positive cases. Since then, immunohistochemistry has been used to facilitate the assessment in many cancers. Nakanishi [31] have used immunohistochemistry for cytokeratin (AE1/AE3) to distinguish cancer cells from inflammatory cells and evaluated tumor budding in thoracic esophageal squamous cell carcinoma. Ohtsuki [32] have shown that pan-cytokeratin stains help identify buds and have improved prognostic value over hematoxylin and eosin stains in colorectal carcinoma. Koelzer [18], in their multicenter interobserver study on colorectal cancer, demonstrated more than three times as many tumor buds with pancytokeratin immunohistochemistry than with hematoxylin and eosin stains, and their results led to marked improvement in interobserver agreement. However, even with these advantages, some authors have argued that immunohistochemical staining may be too costly and timeconsuming to enter routine practice [33]. Although some studies have found immunohistochemistry to be superior to hematoxylin and eosin with regard to reproducibility and interobserver agreement, others have not [34]. van Wyk [35] demonstrated that pan-keratin is neither more reproducible nor gives a higher prognostic value than hematoxylin and eosin scoring in colorectal cancer. Moreover, the ITBCC group recommends that tumor budding should be evaluated on hematoxylin and eosin because the vast majority of outcome data is based on hematoxylin and eosin assessment. In addition, the costeffectiveness of hematoxylin and eosin allows tumor budding to be assessed worldwide [16].

In oral and head and neck squamous cell carcinomas, there are some reports that the immunohistochemical staining of pancytokeratin is helpful to identify epithelial cells when lymphoid infiltration obscures observation, and pan-keratin was more sensitive and easy to score than hematoxylin and eosin in OSCC $[13,36]$ (Figure 1). However, tumor budding can be detected using a hematoxylin and eosin stained slide and is less expensive as in colorectal cancer. In any case, the evaluation method for determining a budding score should be standardized because the results will be different depending on whether immunochemical staining is used to count tumor budding.

\section{Biopsy specimen or resected specimen for assessment of tumor budding}

For assessment of tumor budding from surgical resection 

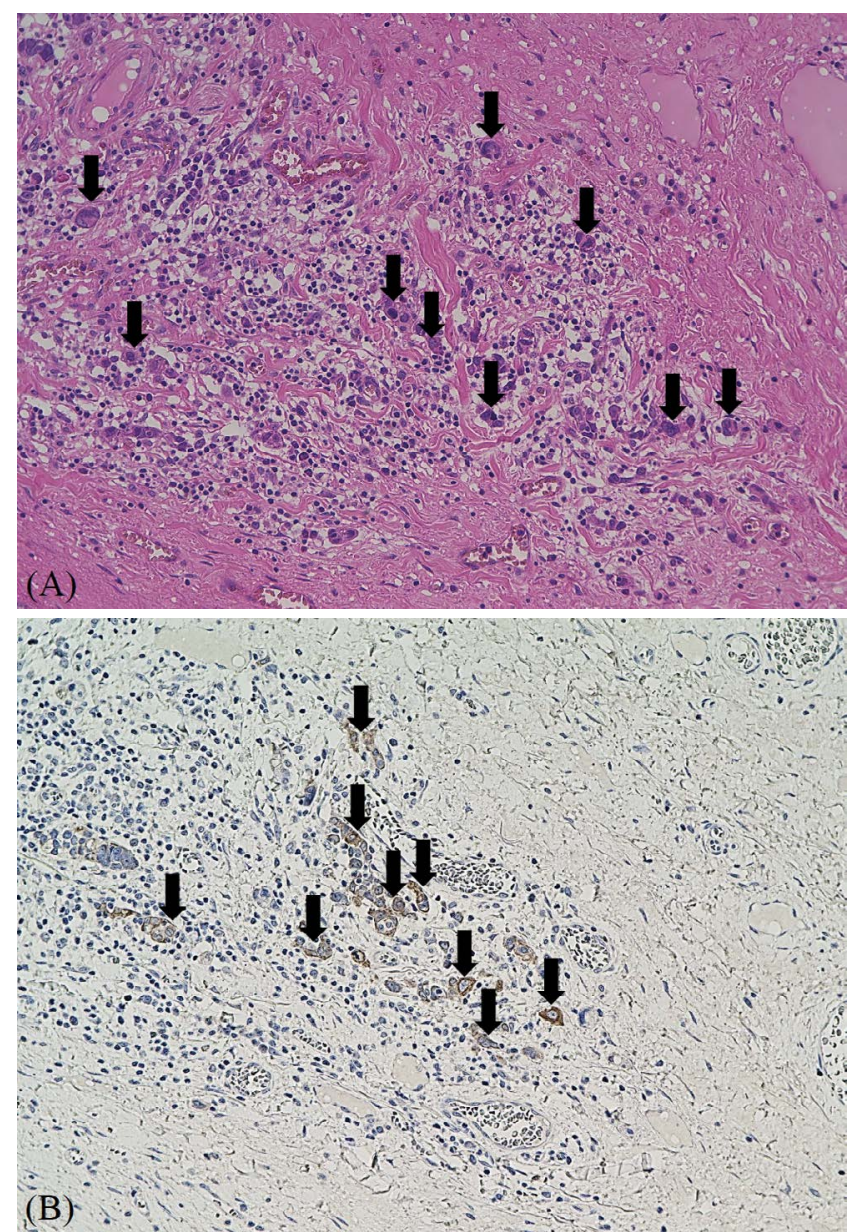

Figure 1: Tumor budding at the invasive front of oral squamous cell carcinoma. H\&E section (A) and immnuno-staining for multi cytokeratin (B) at high magnification.

specimens in colorectal carcinoma, budding activity in preoperative biopsies has been considered. Morodomi [37] showed in 1989 that the presence of budding in biopsies from rectal cancer patients is strong associated with lymphatic invasion and lymph node metastasis. Zlobec [38] reported that the presence of budding cells in an endoscopic biopsy was found to be significantly associated with more advanced T-stage, nodal involvement, and the presence of lymphovascular invasion in the resected specimen. Furthermore, a high level of tumor budding in a biopsy correlated with the presence of distant metastasis. These studies highlight the diagnostic, prognostic, and predictive potential of tumor budding even in biopsy specimens.

In oral and head and neck squamous cell carcinomas, Seki [39] showed that tumor budding evaluated using biopsy specimens was a good predictive factor for lymph node metastasis in squamous cell carcinoma of the tongue and floor of the mouth and was essentially unaffected by infiltrative patterns and tumor depth. In addition, there was a good correlation between biopsies and resected specimens for tumor budding. They also demonstrated that tumor budding in preoperative biopsy specimens is a useful and good predictor of prognosis for relapse-free survival in patients with T1/2 stage OSCC with a tumor depth $<5 \mathrm{~mm}$ and $\mathrm{cN} 0$ cancer. Moreover, tumor budding in biopsy specimens was found to be an independent and powerful predictor of lymph node metastasis and prognosis [40]. These results show that tumor budding score in biopsy specimens is a significant predictive factor for prognosis in early OSCC. However, more and accurate information can be obtained in resected specimens compared to biopsies, and further studies are needed to validate these findings.

\section{Tumor Budding and Epithelial Mesenchymal Transition}

The epithelial mesenchymal transition (EMT) is a biological process that allows a polarized epithelial cell, which normally interacts with the basement membrane via its basal surface, to undergo multiple biochemical changes that enable it to assume a mesenchymal cell phenotype, which includes enhanced migratory capacity, invasiveness, elevated resistance to apoptosis, and increased production of extracellular matrix (ECM) components [41]. EMT is a process whereby tumor cells gain migratory and invasive properties as mesenchymal cells during the cancer pathological process. The activation of the EMT program allows cancer cells to progress and metastasize through induction of cytoskeletal changes, migration potential and the capacity to remodel the extracellular matrix. During EMT, cancer cells loose epithelial characteristics, such as cell polarity and cell-cell adhesion, and gain mesenchymal characteristics and migratory capacity [42]

\section{Discussion}

Therefore, tumor budding may morphologically reflect the process of EMT [43], and the process of tumor budding has been linked to EMT. Budding is associated with loss of cellular adhesion as well as the presence of invasion at the invasive front and has been postulated to be closely associated with EMT [19]. Many reports indicate an association between tumor budding and EMT in colorectal cancer, pancreatic cancer, and esophageal squamous cell carcinoma. EMT is mainly characterized at the molecular level as loss of E-cadherin, gain of $\mathrm{N}$-cadherin, and upregulation of vimentin. Differential expression of several crucial EMT-related genes, including $\beta$-catenin [44], claudins [45], laminin 5, and $\gamma 2$ chains [46], in cancer cells in the invasive tumor front have further confirmed the existence of EMT cells in the invasive tumor front.

In oral and head and neck squamous cell carcinomas, Wang [22] demonstrated that the expression of E-cadherin is significantly reduced in cells located in the invasive tumor front and in cells located in tumor buds, when compared with those located in the central and superficial portions of the tumor samples and observed that reduction in E-cadherin in the invasive tumor front and budding cells was accompanied by an increase in vimentin expression in tongue carcinoma. Attramadal [13] showed that expression of the EMT marker E-cadherin was lost and vimentin was upregulated at the invasive front in T1-2/N0M0 OSCC, which indicates that EMT occurred. However, they did not find loss of keratin expression and general upregulation of $\mathrm{N}$-cadherin, indicating that only partial EMT was present in these early oral carcinomas. These data show that tumor budding was associated with EMT-like changes, and partial EMT, with at least a subset of tumors displaying a true hybrid, single-cell epithelial/mesenchymal cells phenotype in the buds was present in early carcinomas.

The relationship between cancer stem(CSC) cells and EMT, Chen, et al. demonstrated that CSCs have been associated with EMT and tumor buds and are expected to be present in the tumor invasive front [47]. Brabletz suggested that so-called migrating CSCs are frequent in buds in the tumor invasive front [48]. These finding are very important and interesting, but, further investigations should be needed for evaluation [49-54].

\section{Conclusion}

Recently, tumor budding was reported to increase the risk of 
lymph node metastasis and was an independent prognostic factor in several cancers including OSCC. The definition of tumor budding as the presence of individual cancer cells or small clusters (fewer than five cancer cells) at the invasive front of the tumor is generally accepted worldwide and these buds represent a more aggressive and malignant phenotype. However, there is some controversy on the assessment of tumor budding, such as the cut-off point, selecting field, field size, "hot spot" or "10 high-power fields", with or without immunohistochemical staining, and biopsy or resected specimen. The association among the intensity of tumor budding, risk of lymph node metastasis, and poor prognosis in OSCC is strongly supported by studies in this review. However, it remains challenging to standardize a scoring method for budding, and further research is needed.

\section{References}

1. Sankaranarayanan $R$ (1990) Oral cancer in India: A clinical and epidemiological review. Oral Surg Oral Med Oral Pathol 69: 325-330.

2. Llewellyn CD, Johnson NW, Wrnakulasuriya KA (2001) Risk factors for squamous cell carcinoma of the oral cavity in young people: A comprehensive review. Oral Oncol 37: 401-418.

3. Brandwein-Gensler M, Smith RV (2010) Prognostic indicators in head and neck oncology including the new $7^{\text {th }}$ edition of the AJCC staging system. Head and Neck Pathol 4: 53-61.

4. Hinni ML, Ferlito A, Brandwein-Gensler MS, Takes RP, Silver CE, et al. (2012) Surgical margins in head and neck cancer: A contemporary review. Head Neck 35: $1362-1370$

5. Masuda R, Kijima H, Imamura N, Aruga N, Nakamura Y, et al. (2012) Tumor budding is a significant indicator of a poor prognosis in lung squamous cell carcinoma patients. Mol Med Rep 6: 937-943.

6. Teramoto H, Koike M, Tanaka C, Yamada S, Nakayama G, et al. (2013) Tumor budding as useful prognostic marker in T1-stage squamous cell carcinoma of the esophagus. J Surg Oncol 108: 42-46.

7. Prall F, Nizze H, Barten M (2005) Tumor budding as prognostic factor in stage I/II colorectal carcinoma. Histopathology 47: 17-24.

8. Imai T (1954) The growth of human carcinoma: A morphological analysis Fukuoka Igaku Zasshi 45: 72-102.

9. Mc Gavran MH, Bauer WC, Ogura JH (1961) The incidence of cervical lymph node metastases from epidermoid carcinoma of the larynx and their relationship to certain characteristics of the primary tumor: A study based on the clinical and pathological findings for 96 patients treated by primary en bloc laryngectomy and radical neck dissection. Cancer 14: 55-66.

10. Anneroth G, Hansen LS (1984) A methodologic study of histologic classification and grading of malignancy in oral squamous cell carcinoma. Scand J Dent Res 92: $448-468$

11. Yamamoto E, Kohama G, Sunakawa H, Iwai M, Hiratsuka H (1983) Mode of invasion, bleomycin sensitivity, and clinical course in squamous cell carcinoma of the oral cavity. Cancer 51: 2175-2180.

12. Helweg-Larsen K, Graem N, Meistrup-Larsen KI, Meistrup-Larsen U (1978) Clinical relevance of histological grading of cancer of the larynx. Acta Pathol Microbiol Scand 86A: 499-504.

13. Attramadal CG, Kumar S, Boysen ME, Dhakal HP, Nesland JM, et al. (2015) Tumor budding, EMT and cancer stem cells in T1-2/N0 oral squamous cell carcinomas. Anticancer Res 35: 6111-6120.

14. Hase K, Shatney C, Johnson D, Trollope M, Vierra M (1993) Prognostic value of tumor budding in patients with colorectal cancer. Dis Colon Rectum 36: 627635 .

15. Ueno H, Murphy J, Jass JR, Mochizuki H, Talbot IC (2001) Tumor budding as an index to estimate the potential of aggressiveness in rectal cancer Histopathology 40: 127-132.

16. Lugli A, Kirsch R, Ajioka Y, Bosman F, Cathomas G, et al. (2016) Recommendations for reporting tumor budding in colorectal cancer based on the International Tumor Budding Consensus Conference (ITBCC). Mod Patho 30: $1299-1311$
17. Horcic M, Koelzer VH, Karamitopoulou E, Terracciano L, Puppa G, et al. (2013) Tumor budding score based on 10 high-power fields is a promising basis for a standardized prognostic scoring system in stage II colorectal cancer. Hum Pathol 44: 697-705.

18. Koelzer VH, Zlobec I, Berger MD, Cathomas G, Dawson H, et al. (2015) Tumo budding in colorectal cancer revisited: Results of a multicenter interobserver study. Virchows Arch 466: 485-493.

19. Niwa Y, Yamada S, Koike M, Kanda M, Fujii T, et al. (2014) Epithelial to mesenchymal transition correlates with tumor budding and predicts prognosis in esophageal squamous cell carcinoma. J Surg Oncol 110: 764-469.

20. Almangush A, Karhunen M, Hautaniemi S, Salo T, Leivo I (2015) Prognostic value of tumor budding in oesophageal cancer: A meta-analysis. Histopathology 68: 173-182.

21. Marangon Junior $H$, Rocha VN, Leite CF, De Aguiar MC, Souza PE, et al. (2014) Laminin-5 gamma 2 chain expressions is associated with intensity of tumor budding and density of stromal myofibroblasts in oral squamous cell carcinoma. J Oral Pathol Med 43: 199-204.

22. Wang C, Huang H, Huang Z, Wang A, Chen X, et al. (2011) Tumor budding correlates with poor prognosis and epithelial-mesenchymal transition in tongue squamous cell carcinoma. J Oral Pathol Med 40: 545-551.

23. Almangush A, Bello IO, Keski-Säntti H, Mäkinen LK, Kauppila JH, et al. (2014) Depth of invasion, tumor budding, and worst pattern of invasion: Prognostic indicators in early-stage oral tongue cancer. Head Neck 36: 811-818.

24. Karamitopoulou E, Zlobec I, Kölzer V, Kondi-Pafiti A, Patsouris ES, et al. (2013) Proposal for a 10-high-power-fields scoring method for the assessment of tumor budding in colorectal cancer. Mod Pathol 26: 295-301.

25. Xie N, Wang C, Liu X, Li R, Hou J, et al. (2015) Tumor budding correlates with occult cervical lymph node metastasis and poor prognosis in clinical earlystage tongue squamous cell carcinoma. J Oral Pathol Med 44: 266-272.

26. Marangon Junior $H$, Leão PLR, Melo VVM, Caixeta ÂB, Souza PEA, et al. (2017) Cell proliferation is associated with intensity of tumor budding in ora squamous cell carcinoma. J Oral Pathol Med 2: 1.

27. Yamada SI, Otsuru M, Yanamoto S, Hasegawa T, Aizawa H, et al. (2017) Progression level of extracapsular spread and tumor budding for cervical lymph node metastasis of OSCC. Clin Oral Investig 22: 1311-1318.

28. Weichert W, Kossakowski C, Harms A, Schirmacher P, Muley T, et al. (2016) Proposal of a prognostically relevant grading scheme for pulmonary squamous cell carcinoma. Eur Respir J 47: 938-946.

29. Boxberg M, Jesinghaus M, Dorfner C, Mogler C, Drecoll E, et al. (2017) Tumor budding activity and cell nest size determine patient outcome in oral squamous cell carcinoma: Proposal for an adjusted grading system. Histopathology 70 $1125-1137$.

30. Prall F, Nizze H, Barten M (2005) Tumor budding as prognostic factor in stage I/II colorectal carcinoma. Histopathology 47: 17-24.

31. Nakanishi Y, Ohara M, Doumen H, Kimura N, Ishidate T, et al. (2010) Correlation between tumor budding and post-resection prognosis in patients with invasive squamous cell carcinoma of the thoracic esophagus. World J Surg 35: 349-356.

32. Ohtsuki K, Koyama F, Tamura T, Enomoto Y, Fujii H, et al. (2008) Prognostic value of immunohistochemical analysis of tumor budding in colorecta carcinoma. Anticancer Res 28: 1831-1836.

33. Wang LM, Kevans D, Mulcahy H, O'Sullivan J, Fennelly D, et al. (2009) Tumo budding is a strong and reproducible prognostic marker in T3N0 colorectal cancer. Am J Surg Pathol 33: 134-141.

34. Kai K, Aishima S, Aoki S, Takase Y, Uchihashi K, et al. (2016) Cytokeratin immunohistochemistry improves interobserver variability between unskilled pathologists in the evaluation of tumor budding in T1 colorectal cancer. Pathol Int 66: 75-82.

35. Van Wyk HC, Park J, Roxburgh C, Horgan P, Foulis A, et al. (2015) The role of tumor budding in predicting survival in patients with primary operable colorectal cancer: A systematic review. Cancer Treat Rev 41: 151-159.

36. Almangush A, Coletta RD, Bello IO, Bitu C, Keski-Säntti H, et al. (2015) A simple novel prognostic model for early stage oral tongue cancer. Int $\mathrm{J}$ Oral Maxillofac Surg 44: 143-150.

37. Morodomi T, Isomoto H, Shirouzu K, Kakegawa K, Irie K, et al. (1989) An index for estimating the probability of lymph node metastasis in rectal cancers. Lymph 
Citation: Kirita T, Yamakawa N, Ueda N, Yagyuu T (2018) Tumor Budding as a Useful Prognostic Indicator in Early Oral Squamous Cell Carcinoma. J Cancer Sci Ther 10: 162-167. doi: 10.4172/1948-5956.1000536

node metastasis and the histopathology of actively invasive regions of cancer. Cancer 63: 539-543.

38. Zlobec I, Hädrich M, Dawson H, Koelzer VH, Borner M, et al. (2013) Intratumoral budding (ITB) in preoperative biopsies predicts the presence of lymph node and distant metastases in colon and rectal cancer patients. $\mathrm{Br} \mathrm{J}$ Cancer 110: 1008-1013.

39. Seki M, Sano T, Yokoo S, Oyama T (2015) Histologic assessment of tumor budding in preoperative biopsies to predict nodal metastasis in squamous cell carcinoma of the tongue and floor of the mouth. Head Neck 38: 1582-1590.

40. Seki M, Sano T, Yokoo S, Oyama T (2017) Tumor budding evaluated in biopsy specimens is a useful predictor of prognosis in patients with cNO early stage oral squamous cell carcinoma. Histopathology 70: 869-879.

41. Kalluri R (2009) EMT: When epithelial cells decide to become mesenchymallike cells. J Clin Invest 119: 1417-1419.

42. De Smedt L, Palmans S, Sagaert $X$ (2015) Tumor budding in colorectal cancer: What do we know and what can we do? Virchows Arch 468: 397-408.

43. Guarino M, Rubino B, Ballabio G (2007) The role of epithelial-mesenchymal transition in cancer pathology. Pathology 39: 305-318.

44. Wang X, Zhang J, Fan M, Zhou Q, Deng H, et al. (2009) The expression of $\mathrm{E}$-cadherin at the invasive tumor front of oral squamous cell carcinoma: Immunohistochemical and RT-PCR analysis with clinicopathological correlation. Oral Surg Oral Med Oral Pathol Oral Radiol Endod 107: 547-554.

45. Bello IO, Vilen ST, Niinimaa A, Kantola S, Soini Y, et al. (2008) Expression of claudins $1,4,5$ and 7 and occludin, and relationship with prognosis in squamous cell carcinoma of the tongue. Hum Pathol 39: 1212-1220.

46. Franz M, Richter P, Geyer C, Hansen T, Acuña LD, et al. (2007) Mesenchymal cells contribute to the synthesis and deposition of the laminin- 5 gamma-2 chain in the invasive front of oral squamous cell carcinoma. J Mol Histol 38: 183-190.

47. Chen C, Zimmermann M, Tinhofer I, Kaufmann AM, Albers AE (2013) Epithelial-to-mesenchymal transition and cancer stem (like) cells in head and neck squamous cell carcinoma. Cancer Lett 338: 47-56.

48. Brabletz T, Jung A, Spaderna S, Hlubek F, Kirchner T (2005) Opinion: Migrating cancer stem cells-An integrated concept of malignant tumor progression. Nat Rev Cancer 5: 744-749.

49. Sarioglu S, Acara C, Akman FC, Dag N, Ecevit C, et al. (2010) Tumor budding as a prognostic marker in laryngeal carcinoma. Pathol Res Pract 206: 88-92.

50. Luo WR, Gao F, Li SY, Yao KT (2012) Tumor budding and the expression of cancer stem cell marker aldehyde dehydrogenase 1 in nasopharyngeal carcinoma. Histopathology 61: 1072-1081.

51. Angadi PV, Patil PV, Hallikeri K, Mallapur MD, Hallikerimath S, et al. (2015) Tumor budding is an independent prognostic factor for prediction of lymph node metastasis in oral squamous cell carcinoma. Int J Surg Pathol 23: 102-110.

52. Sawazaki-Calone I, Rangel A, Bueno AG, Morais CF, Nagai HM, et al. (2015) The prognostic value of histopathological grading systems in oral squamous cell carcinomas. Oral Dis 21: 755-761.

53. Jensen DH, Dabelsteen E, Specht L, Fiehn AM, Therkildsen MH, et al. (2015) Molecular profiling of tumor budding implicates TGF $\beta$-mediated epithelialmesenchymal transition as a therapeutic target in oral squamous cell carcinoma. J Pathol 236: 505-516.

54. Pedersen NJ, Jensen DH, Lelkaitis G, Kiss K, Charabi B, et al. (2017) Construction of a pathological risk model of occult lymph node metastases for prognostication by semi-automated image analysis of tumor budding in earlystage oral squamous cell carcinoma. Oncotarget 8: 18227-18237. 